\title{
Modélisation du soudage par point : définition des conditions interfaciales et validation expérimentale
}

\author{
Éric Feulvarch ${ }^{1,2, a}$, Philippe Rogeon ${ }^{3}$, Patrick Carré ${ }^{3}$, Gauthier Sibilia ${ }^{4,5}$ et \\ JeAN-Michel BergheaU ${ }^{1}$ \\ 1 Laboratoire de Tribologie et Dynamique des Systèmes, UMR 5513 CNRS/ECL/ENISE, École Nationale d'Ingénieurs \\ de Saint-Étienne, 58 rue Jean Parot, 42023 Saint-Étienne Cedex 2, France \\ 2 ESI Group, Le Récamier, 70 rue Robert, 69458 Lyon Cedex 06, France \\ 3 Laboratoire d'Études Thermiques Énergétiques et Environnement, Université de Bretagne Sud, Rue de Saint-Maudé, \\ BP 92116, 56321 Lorient Cedex 3, France \\ 4 Laboratoire Génie des Matériaux, École Polytechnique de l'Université de Nantes, La Chantrerie, Rue Christian Pauc, \\ BP 50609, 44306 Nantes Cedex 3, France \\ 5 PSA Peugeot Citroën, Centre Technique de Vélizy, MXP/CEB/ASG, Route de Gisy, 78943 Vélizy Villacoublay Cedex, France
}

Reçu le 1 avril 2004, accepté le 4 avril 2006

\begin{abstract}
Résumé - Cet article présente un modèle de connaissance pour mieux comprendre et expliquer les mécanismes à l'origine de la formation de la soudure dans le procédé de soudage par point en nous limitant ici au cas d'assemblages symétriques de tôles nues en acier extra-doux. Dans une première approche, on considère un modèle simplifié prenant en compte les couplages entre les phénomènes électrique, thermique et métallurgique dans l'assemblage mais en supposant les surfaces de contact constantes aux interfaces électrode-tôle et tôle-tôle. Dans une seconde approche le modèle est complété par un calcul mécanique qui permet de calculer les évolutions des surfaces de contact. Les résistances de contact électrique et thermique sont mesurées en fonction de la température et de la pression sur un dispositif expérimental conçu à cet effet. La variation des profils des électrodes en fonction du nombre de soudures réalisées et ses conséquences sur les surfaces de contact sont de même présentées. Le modèle numérique est validé en comparant, d'une part, la taille et la forme des points soudés relevées expérimentalement et calculées numériquement et, d'autre part, les cinétiques thermiques mesurées et calculées dans les électrodes et les tôles. Les techniques d'instrumentation mises au point visent à limiter les perturbations inévitables générées par l'intrusion des capteurs. Les hypothèses et les jeux de données considérés dans le modèle pour définir les conditions interfaciales sont enfin discutés.
\end{abstract}

Mots clés : soudage par points / mesure de résistance électrothermique de contact / modélisation éléments finis

\begin{abstract}
Modeling of spot welding: definition of the interfacial contact conditions and experimental validation. In this paper, we present a numerical model for better understanding and explaining the weld growth mechanisms of spot welds. In this way, only simple configurations of symmetrical uncoated steel sheet assemblies are considered. In a first approach, a simplified model is defined, assuming constant contact surfaces at the interfaces but taking account of the coupled electrical, thermal and metallurgical phenomena in the assembly. In a second approach, the model is supplemented by a mechanical calculation which makes possible to take into account the evolutions of the contact surfaces. Electrical and thermal contact resistance evolutions are measured according to temperature and pressure by using an experimental device developed for this purpose. The modification of the electrodes profile according to the number of welds carried out and its consequences on the contact surfaces are also presented. Finally numerical models are validated by comparing calculated and measured thermal kinetics in electrodes and sheets. The experimental technique developed here aims at prevent the disturbances generated by the sensors. The assumptions and the data considered in the numerical models to define the interfacial conditions are then discussed.
\end{abstract}

Key words: spot welding / electrothermal contact resistance measurements / finite element simulation

a Auteur correspondant : eric.feulvarch@esi-group.com 


\section{Introduction}

Le soudage par résistance par points est un procédé courant dans l'industrie automobile en raison de sa capacité à assembler des tôles minces. D'un point de vue industriel, l'introduction de nouvelles familles d'acier (en particulier d'aciers à haute limite élastique) remet en question les référentiels de soudage classiques. Les conditions dans lesquelles se réalise la soudure par point sont très défavorables à l'observation et à la mesure (temps très court de l'ordre de $0,2 \mathrm{~s}$ et formation du point soudé en aveugle). C'est la raison pour laquelle, en partenariat avec PSA Peugeot Citroën, la simulation numérique a été envisagée comme outil de prédiction pour modéliser le développement d'un point de soudure, sa forme, sa position dans l'assemblage, ainsi que les phases métallurgiques constitutives.

Du point de vue physique, l'étude théorique d'un tel procédé est un problème complexe qui fait intervenir plusieurs phénomènes tels que l'électrocinétique, la thermique, la métallurgie et la mécanique. Un certain nombre d'études s'accordent sur la complexité des couplages physiques [1-3], de même que sur le rôle essentiel des conditions de contact entre électrode et tôle et entre tôle et tôle. Pour caractériser les conditions interfaciales nous avons, d'une part, développé un dispositif de mesure de résistance de contact électrique et thermique en fonction de la température et de la pression, et d'autre part, étudié la modification du profil de la face active de l'électrode en fonction du nombre de soudures réalisées.

Nous avons également développé des techniques d'instrumentation d'assemblages en capteurs de température. En plus des coupes macroscopiques habituellement utilisées pour la validation de tels modèles, les signaux thermiques mesurés dans les électrodes et les tôles sont également confrontés à nos modèles numériques.

Enfin, nous avons développé deux modèles numériques. Le premier modèle, appelé modèle simplifié, suppose que les surfaces de contact sont constantes. Dans le second modèle, le couplage avec les aspects mécaniques est intégré et permet de calculer les évolutions des surfaces de contact durant les différentes phases du procédé (accostage, soudage et forgeage). Pour ce faire, une formulation numérique du contact électrothermique est proposée. Pour faciliter la compréhension, nous avons restreint l'étude à des assemblages symétriques de tôles nues et défini le cas académique de la tôle équivalente d'épaisseur double. En supprimant l'interface tôle-tôle les effets des deux interfaces peuvent ainsi être découplés.

\section{Principe du soudage par points}

L'opération de soudage par points se décompose en trois phases fondamentales (Fig. 1a), l'accostage, le soudage et le forgeage, suivies d'une phase de refroidissement des tôles soudées à l'air libre. Le point soudé est composé d'un noyau fondu (Zone Fondue, ZF) entouré d'une Zone Affectée Thermiquement (ZAT) (Fig. 1b).

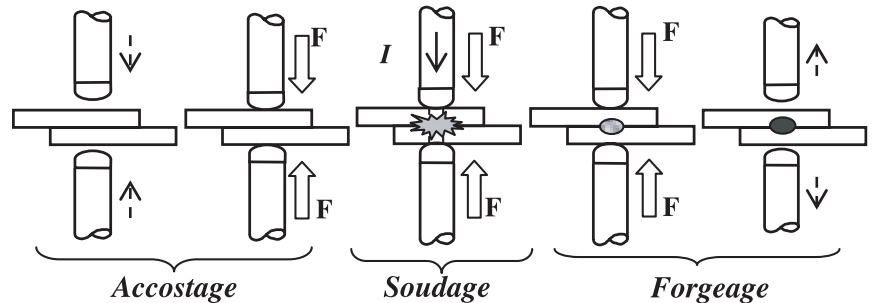

(a)

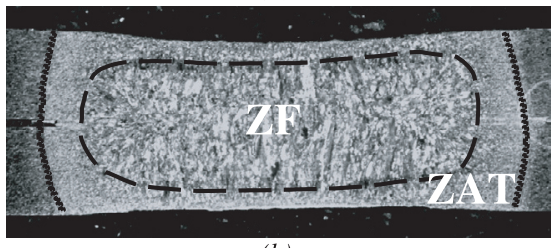

(b)

Fig. 1. (a) Principe du soudage par résistance. (b) Exemple de zone fondue.

L'échauffement qui conduit à la création du noyau fondu résulte de la génération de chaleur par effet Joule dans les différentes résistances qui constituent l'assemblage soudé : les résistances intrinsèques des tôles et les différentes résistances de contact électrique qui apparaissent au niveau des interfaces électrode-tôle et tôle-tôle. Les résistances de contact dépendent de mécanismes qui se situent à deux échelles différentes. Les surfaces apparentes de contact, de part leur dimension, vont influer directement sur la macroconstriction du courant électrique à l'échelle du procédé, tandis que les défauts de contact (rugosités, ondulations) vont générer des microconstrictions des lignes de courant électrique responsables en partie de la résistance de contact. Ces deux effets combinés expliquent le rôle essentiel des interfaces dans le procédé de soudage par point. La variation des surfaces de contact en cours de soudage va donc influer directement sur la formation du point soudé. Par conséquent, une bonne connaissance des valeurs des résistances de contact RC (électrique, $R C E$ et thermique, $R C T$ ) constitue un préalable à toute modélisation pertinente du soudage par point [4].

Les résistances de contact électriques vont jouer essentiellement un rôle d'étincelle qui amorce le processus d'auto-échauffement de l'assemblage par l'augmentation rapide des résistances intrinsèques des tôles. Les résistances de contact thermique constituent quant à elles des barrières thermiques : par exemple celle à l'interface électrode-tôle favorise l'échauffement des tôles en les isolant des électrodes en cuivre thermostatées.

\section{Caractérisation des résistances de contact}

Un dispositif de mesure des résistances de contact électrique et thermique en fonction de la température, sous forte pression, a été conçu et développé au Laboratoire d'Études Thermiques Énergétiques et Environnement (LET2E), de l'Université de Bretagne Sud de Lorient. Nous avons envisagé une technique de mesure 
ex situ intermédiaire entre celles de Babu et al. [5] et de Vogler et Sheppard [6].

Ces échantillons sont placés dans une presse, entre deux poinçons par lesquels est amené le courant électrique de mesure. Aux bornes des échantillons deux électrodes instrumentées servent à la mesure de la différence de potentiel. Contrairement aux auteurs précités, on intègre dans la mesure de résistance un nombre variable $n$ de contacts. On utilise pour cela une technique d'empilement d'échantillons dont l'objectif est d'amplifier le signal. Pour l'étude des deux types de contact tôle/tôle et électrode/tôle, les empilements sont constitués respectivement d'échantillons en acier ou de couples d'échantillons acier/cuivre allié (matériaux de l'étude).

Dans le cas de l'étude du contact tôle/tôle, la résistance globale de l'empilement mesurée avec $n+$ 1 échantillons résulte de l'addition d'une résistance initiale $R 0$, constituée des deux électrodes et du premier échantillon, de $n$ résistances de contact $R C E$ et de $n$ résistances ohmiques $R_{\mathrm{O}}$. Chaque échantillon supplémentaire introduit dans l'empilement une résistance ohmique et une résistance de contact additionnelle. Ainsi, il est possible d'exprimer la résistance globale de l'empilement lors de la $n^{\text {ème }}$ mesure en fonction de $n, R 0, R C E$ et $R_{\mathrm{O}}$ selon :

$$
R n=p \times n+R 0
$$

où,

$$
p=R C E(T / T)+R_{\text {O_acier }}
$$

Dans le cas de l'étude du contact électrode/tôle, la simple différence tient à ce qu'à chaque mesure un couple d'échantillons neufs est ajouté. La résistance globale de l'empilement lors de la $n^{\text {ème }}$ mesure peut alors s'écrire selon l'équation (1) avec toutefois :

$$
p=2 \times R C E(E / T)+\left(R_{\text {O_acier }}+R_{\text {O_cuivre }}\right)
$$

Dans les deux cas, le tracé de la résistance d'empilement $R n$ en fonction du nombre $n$ d'échantillons ou de couples d'échantillons, permet de déterminer $R C E$ à partir de la pente $p$ de la droite obtenue, sans faire intervenir la résistance initiale $R 0$ qui contient toutes les résistances parasites de l'empilement.

Le dispositif expérimental, le protocole de préparation des échantillons ainsi que la méthodologie de mesure sont décrits en détail dans la thèse de G. Sibilia [7].

\subsection{Résistance de contacts électrique tôle/tôle}

Dans les conditions de soudage les interfaces sont soumises à des dynamiques thermiques extrêmes (plusieurs milliers de degrés Celsius par seconde) et l'interface électrode-tôle à des gradients thermiques élevés (plusieurs centaines de degrés Celsius). On a voulu dans le dispositif expérimental étudier l'effet du fluage ou de l'oxydation potentielle des surfaces en faisant varier la durée de la montée en température : une montée lente en $4 \mathrm{~h}$ par palier de température jusqu'à $450^{\circ} \mathrm{C}$ et une montée plus

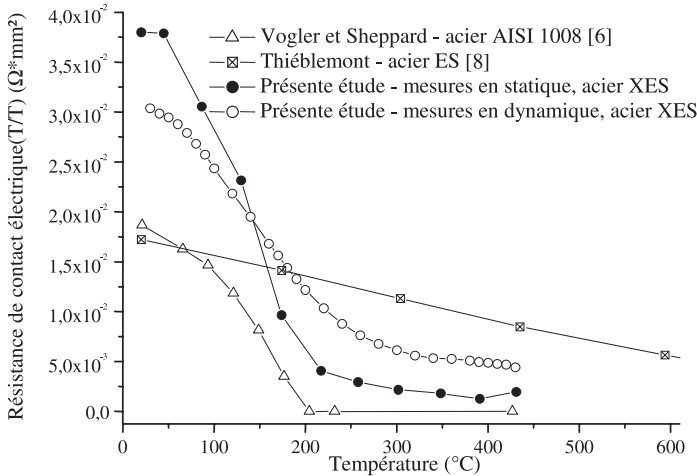

Fig. 2. Courbes $R C E$ (tôle/tôle) $=f(T)$ - cas des tôles nues pour les deux campagnes d'essais et confrontation à la littérature.

rapide en $4 \mathrm{~min}$ (mesures en statique et en dynamique (Fig. 2)).

À partir des évolutions des résistances d'empilement avec la température, l'évolution de $R C E$ (tôle/tôle) est déduite par le calcul de la pente $p$ de l'équation (3) pour les différentes températures.

Nous constatons en particulier un écroulement rapide de $R C E$ avec la température : à $250{ }^{\circ} \mathrm{C}$, la valeur a déjà chuté d'un facteur 4 . Cette forte diminution est confirmée par les mesures de Vogler et Sheppard pour des températures inférieures à $200^{\circ} \mathrm{C}$, mais elle ne se retrouve pas dans l'évolution déduite par Thiéblemont [8].

Pour les contacts entre tôles nues, à température ambiante et même sous forte pression, il est établi que le taux de contact réel n'excède pas quelques \% de la surface apparente [9]. Lorsque la température augmente, les propriétés mécaniques du matériau (limite élastique, module d'Young... ) baissent tandis que la résistivité électrique augmente. Ces deux variations ont des effets antagonistes et agissent de façon contradictoire sur $R C E$. Pour les contacts entre tôles nues, le facteur dominant semble être l'augmentation du taux de contact qui conduit à une diminution radicale de $R C E$ avec la température.

\subsection{Résistance de contacts électrique électrode/tôle}

La variation de $R C E$ (électrode/tôle) est représentée sur la figure 3 et confrontée aux résultats de la littérature. $\mathrm{Au}$ chauffage, comme pour le contact de type tôle/tôle, on note une décroissance continue, d'abord rapide puis moins marquée. Comme dans le cas de $R C E$ (tôle/tôle), compte tenu de la dispersion expérimentale, nous constatons que nos résultats sont assez proches de ceux de Vogler et Sheppard mais plus éloignés de ceux de Thiéblemont. Pendant la phase de refroidissement, l'irréversibilité des phénomènes interfaciaux (plastification des aspérités) se manifeste par une hystérésis fortement marquée. 


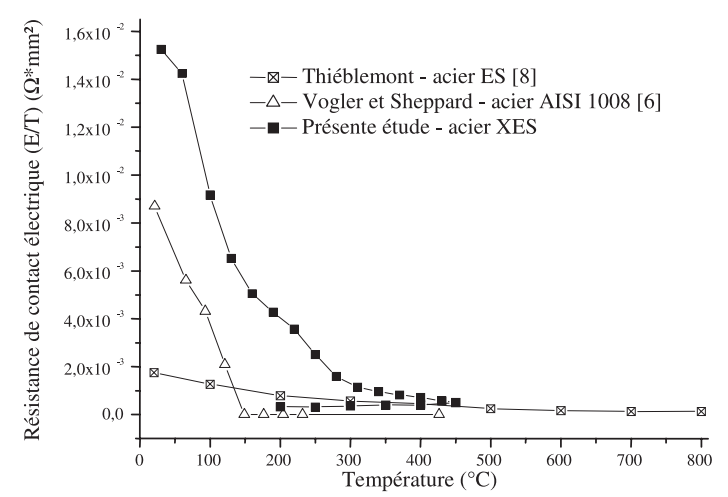

Fig. 3. Courbes $R C E$ (électrode/tôle) $=f(T)$ - cas des tôles nues pour les deux campagnes d'essais et confrontation à la littérature.

\subsection{Résistance de contacts thermique électrode/tôle}

Nous considérons uniquement des configurations de soudage symétriques. Par conséquent, nos mesures se limitent au contact électrode/tôle (couples cuivre/acier).

L'objectif est ici de caractériser les résistances de contact thermiques $R C T$ pour des interfaces dissymétriques en nature, sous de fortes pressions $(>20 \mathrm{MPa})$ et en fonction de la température. Très peu d'études $[8,10]$ font état de mesures dans de telles conditions, plus encore dans le cas du procédé de soudage par point.

Pour déterminer les résistances de contact thermiques, nous avons choisi de développer une méthodologie et un dispositif de mesure spécifique analogues à ceux employés pour les mesures de résistances de contact électriques.

De façon similaire au dispositif expérimental utilisé pour les mesures de $R C E$, nous adaptons le montage constitué des deux poinçons en acier inoxydable instrumentés en thermocouples fins $(80 \mu \mathrm{m})$ entre les mors d'une presse. Pour les raisons évoquées au cours des mesures électriques, on utilise le principe d'empilement d'échantillons de cuivre et d'acier. La technique repose sur la circulation d'un flux de chaleur conservatif $\Phi$ au travers de cet empilement. La génération du flux est assurée par un chauffage dissymétrique, grâce à des colliers chauffants équipant les poinçons.

Les empilements sont constitués d'au moins deux tôles de cuivre et d'une tôle d'acier (résistance $R 0$ ). On ajoute successivement $n$ couples d'échantillons cuivre/acier, qui introduisent chacun dans l'empilement deux résistances de contact thermiques additionnelles. La résistance ther-

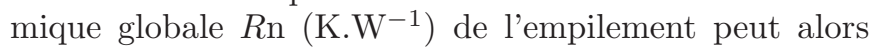
s'exprimer en fonction de $n, R 0, R C T$ et des résistances thermiques $R_{\mathrm{T}}$ des différents échantillons de cuivre et d'acier :

$$
R n=p \times n+R 0
$$

où,

$$
p=2 \times R C T+\left(R_{\mathrm{T}_{\text {_acier }}}+R_{\mathrm{T} \_ \text {cuivre }}\right)
$$

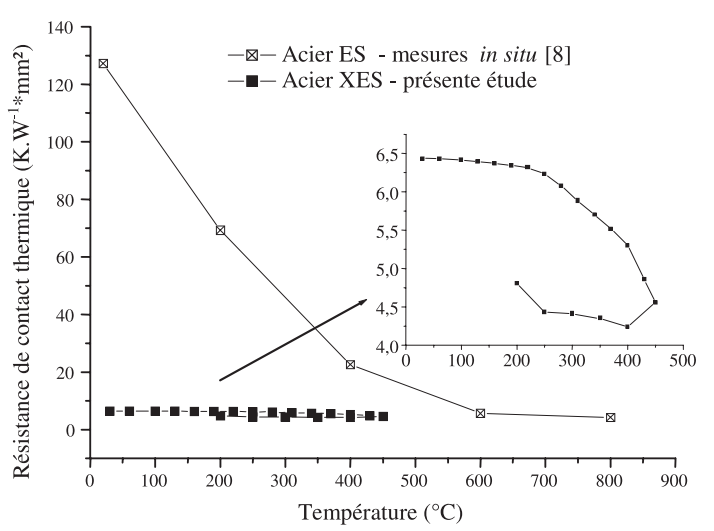

Fig. 4. Courbe $R C T$ (électrode/tôle) $=f(T)$ - cas des tôles XES et confrontation à la littérature.

Par ailleurs, si $\theta_{1}$ et $\theta_{2}$ représentent les températures aux bornes de l'empilement, la résistance thermique globale de l'empilement vaut :

$$
R n=\frac{\theta_{1}-\theta_{2}}{\Phi}
$$

La difficulté de cette mesure réside dans la bonne conservation du flux de chaleur à la traversée de l'empilement. Pour réduire les fuites thermiques latérales, en plus d'un manteau en fibres de céramique (Kerlane ${ }^{\circledR}$ ), un dispositif de garde thermique constitué par un troisième collier chauffant a été adapté.

La variation de $R C T$ avec la température pour le contact XES/cuivre (Fig. 4) est comparée aux valeurs de Thiéblemont. La confrontation montre à nouveau des différences significatives.

Par contre nos résultats apparaissent proches des valeurs estimées par méthodes inverses données dans la thèse de Le Meur [10]. Enfin, à l'image des résistances électriques, nous relevons une hystérésis importante dans l'évolution de $R C T$ entre la phase de chauffage et celle de refroidissement. L'échauffement du contact, maintenu sous forte pression, provoque une déformation plastique permanente des interfaces. Ce résultat se retrouve d'ailleurs très bien dans les travaux de Manach [11], mais aussi dans l'étude de Vogler et Sheppard, dans le cadre de mesures électriques [6].

\section{4 Étude profilométrique}

La variation des surfaces de contact en cours de soudage influe directement sur la formation du point soudé. La littérature montre en outre le rôle prépondérant que jouent le profil et l'état d'usure de la face active des électrodes dans cette évolution [12].

Les deux types d'électrodes de soudage utilisées dans le cadre de cette étude sont des embouts usinés dont la face active est une calotte sphérique de $6 \mathrm{~mm}$ et de $8 \mathrm{~mm}$ de diamètre. Leur rayon de courbure vaut initialement, 40 et $50 \mathrm{~mm}$ respectivement. Cette géométrie spécifique 


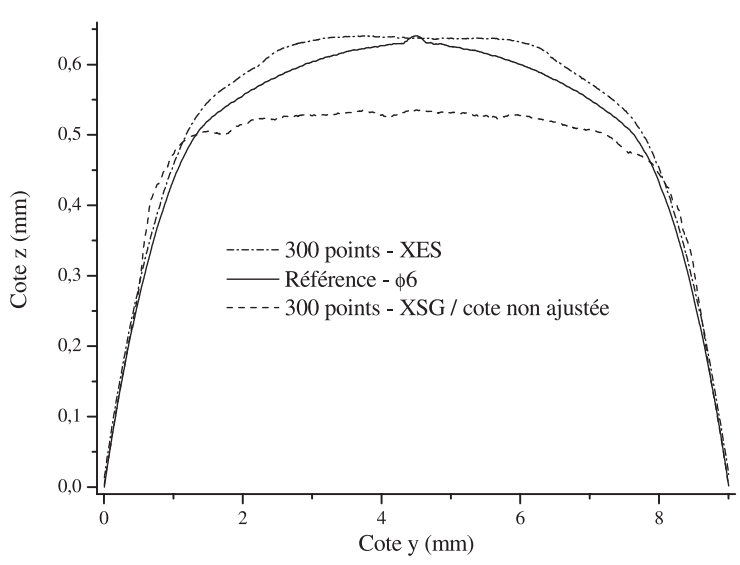

Fig. 5. Variation du profil de l'électrode $\Phi 6$.

permet, en particulier, d'éviter les problèmes d'alignement lors de l'accostage des électrodes sur les pièces à souder.

Toutefois, avec la répétition des soudures, divers phénomènes concourent à la dégradation de leur extrémité (pollution et érosion du profil, [13-15]). Pour pallier cette évolution continue, le référentiel de soudage par points de PSA préconise le recours à une incrémentation du courant de soudage par paliers, et le rodage des électrodes au bout d'un certain nombre de points soudés.

Concernant notre modèle, il est important de considérer un profil d'électrode suffisamment représentatif du cas industriel. Pour ce faire, nous présentons dans cette section la caractérisation expérimentale de la modification du profil des électrodes au cours de leur utilisation.

\subsection{Dispositif expérimental et campagne d'essais}

Les différents facteurs susceptibles d'influer sur la modification de la face active des électrodes sont dans notre cas, le matériau, le type d'électrode $\Phi 6$ ou $\Phi 8$ et le mode de soudage associé (normal ou pulsé). Les paramètres de soudage ainsi que la loi d'incrémentation du courant sont définis selon la norme [16]. Le principe de cette campagne de mesures repose sur la réalisation d'un nombre donné $n$ de points soudés et l'analyse du jeu d'électrodes utilisées pour ces $n$ soudures. La fréquence des mesures est définie selon $n=1,3,5,10,25,50,75,100,150$ et 300 .

La solution technique retenue pour quantifier l'évolution de la face active des électrodes s'est portée sur une méthode à palpeur mécanique, solidaire d'un capteur inductif : les essais ont été réalisés au Laboratoire de Métrologie de Sochaux de PSA.

\subsection{Résultats}

Nous observons une évolution du profil initial, rayonné, vers un aplanissement partiel de la face active de l'électrode dans le cas du soudage des tôles nues

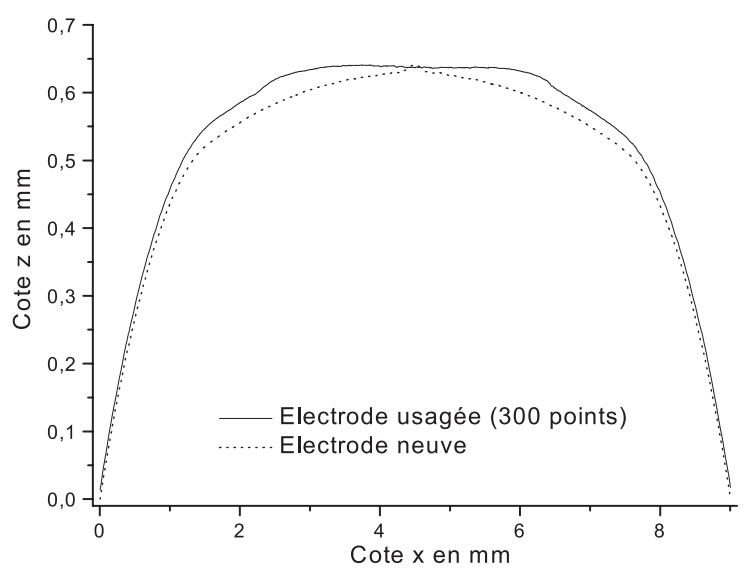

Fig. 6. Variation du profil de l'électrode $\Phi 8$.

en acier extra-doux. Au bout de cinquante soudures au plus, la face active est partiellement plane (Figs. 5 et 6 ). Le profil d'électrode usagée obtenu est aussi comparé avec celui correspondant à une électrode ayant soudé des tôles revêtues (Fig. 5) : on constate dans ce dernier cas le rôle essentiel du revêtement de la tôle sur l'usure de l'électrode, conduisant à un aplanissement global du profil.

Pour étudier l'impact du profil de l'électrode sur les surfaces de contact, la phase d'accostage a été simulée en considérant des profils d'électrodes neuves et usagées. Les résultats numériques révèlent que l'érosion du profil entraîne une augmentation notable des surfaces de contact : par exemple sur un assemblage de tôles de $1 \mathrm{~mm}$ d'épaisseur soudées avec des électrodes (face active $\Phi 6 \mathrm{~mm})$, le rayon de la surface de contact électrode/tôle augmente de 1,8 à $2,45 \mathrm{~mm}$ et le rayon de la surface de contact tôle/tôle passe de 1,8 à 2,7 mm, en passant d'une électrode neuve à une électrode usagée.

\section{Modélisation axisymétrique}

Dans la première approche, seuls les phénomènes électriques, thermiques et métallurgiques sont considérés et la face active des électrodes est plate c'est-à-dire que la géométrie et, plus particulièrement, les surfaces de contact restent constantes au cours du soudage. Dans la seconde approche, un couplage avec la mécanique permet de modéliser l'évolution des surfaces de contact pour des électrodes neuves dont la face active présente une géométrie sphérique. L'objectif d'une telle démarche est de retranscrire l'évolution des surfaces de contact durant la formation de la soudure $[1,2,17-20]$ (dilatation des matériaux, ouverture des tôles en périphérie de l'interface tôle/tôle, création de la zone fondue, indentation des électrodes dans les tôles...).

On a enfin défini le « cas académique » du soudage de la tôle équivalente d'épaisseur double, qui permet en supprimant l'interface tôle/tôle de découpler les effets des deux interfaces électrode/tôle et tôle/tôle. 


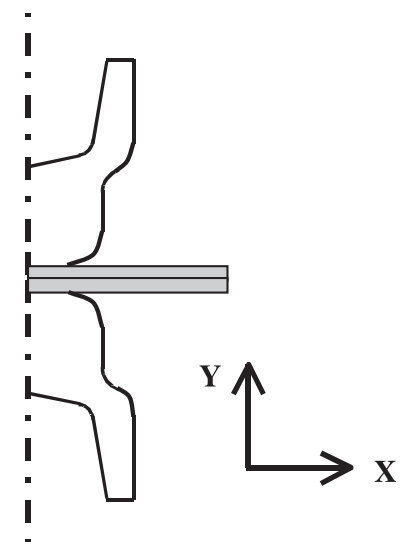

Fig. 7. Représentation schématique de la géométrie axisymétrique et bidimensionnelle.

\subsection{Modèle électro-thermo-métallurgique}

Le modèle que nous proposons se limite aux aspects électriques, thermiques et métallurgiques. Notre objectif est de pouvoir prédéterminer l'emplacement, la taille et la forme du point soudé (zone fondue et zone affectée thermiquement), les cinétiques thermiques dans l'assemblage ainsi que les phases métallurgiques constitutives de la ZAT.

Les principaux résultats des simulations numériques sont la distribution de la densité de courant, les champs thermiques et les proportions de phases métallurgiques.

Compte tenu de la géométrie de révolution du procédé, nous nous limitons à une modélisation bidimensionnelle axisymétrique (Fig. 7).

Dans ce procédé, les fortes interactions entre les phénomènes électriques et thermiques nécessitent le recours à un couplage fort [21]. Le code SYSWELD ${ }^{\circledR}[22]$ permet de résoudre simultanément les équations décrivant les phénomènes électrocinétiques, thermiques et les cinétiques de transformation de phase.

\subsubsection{Modèle électrothermique}

Le problème aux dérivées partielles qui régit les couplages électrothermiques dans un domaine $\Omega$ de frontière $\partial \Omega$ est constitué par la juxtaposition du problème aux dérivées partielles associé à la diffusion de la chaleur et du problème associé aux phénomènes électriques.

Le courant de forte intensité est généralement à la fréquence $50 \mathrm{~Hz}$. De ce fait, les effets dynamiques sont négligeables et les courants électriques peuvent être déterminés à partir d'un modèle électrocinétique [8]. De plus, plusieurs auteurs ont déjà montré qu'il était possible d'utiliser un courant continu équivalent [7,23].
Par conséquent, le champ de potentiel électrique $V(\vec{x})$ et le champ de température $\theta(\vec{x})$ sont solution des équations aux dérivées partielles suivantes :

$$
\begin{gathered}
\operatorname{div}(\sigma(\theta) \cdot \overrightarrow{\operatorname{grad}}(V))=0 \\
\left\{\sum_{\text {phases }} p_{i} \cdot \rho_{i}^{\mathrm{vol}}(\theta) \cdot \frac{\partial H_{i}(\theta)}{\partial t}+\sum_{\text {phases }} \frac{\partial p_{i}}{\partial t} \cdot \rho_{i}^{\mathrm{vol}}(\theta) \cdot H_{i}(\theta)\right\}- \\
\operatorname{div}\left(\sum_{\text {phases }} p_{i} \cdot \lambda_{i}(\theta) \cdot \overrightarrow{\operatorname{grad}}(\theta)\right)-\overrightarrow{\operatorname{grad}}(V) \cdot \sigma(\theta) \cdot \overrightarrow{\operatorname{grad}}(V)
\end{gathered}
$$

avec les conditions aux limites :

$$
\begin{aligned}
& V=V_{\mathrm{d}} \text { sur } \partial \Omega_{V} \\
& \sigma \cdot \overrightarrow{\operatorname{grad}}(V) \cdot \vec{n}=j \text { sur } \partial \Omega_{j} \text { avec } \partial \Omega=\partial \Omega_{V} \cup \partial \Omega_{j} \\
& \theta=\theta_{\mathrm{d}} \quad \operatorname{sur} \quad \partial \Omega_{\theta} \\
& \lambda \cdot \overrightarrow{\operatorname{grad}}(\theta) \cdot \vec{n}=q \text { sur } \partial \Omega_{q} \text { avec } \partial \Omega=\partial \Omega_{\theta} \cup \partial \Omega_{q}
\end{aligned}
$$

Pour chaque phase métallurgique présente en proportion $p_{i}$, la conductivité thermique $\lambda_{i}$, la masse volumique $\rho_{i}$ et l'enthalpie $H_{i}$ dépendent de la température tout comme la conductivité électrique $\sigma$.

Dans le cadre d'une étude de sensibilité [7], G. Sibilia a démontré l'influence négligeable sur les cinétiques thermiques dans l'assemblage et sur la taille de noyau, des échanges thermiques entre les tôles et l'air ambiant d'une part et entre les électrodes et l'eau de refroidissement d'autre part. Par conséquent, tous les échanges thermiques convecto-radiatifs entre l'assemblage et l'extérieur sont négligés en considérant que les parois sont adiabatiques $(q=0)$.

\subsubsection{Conditions interfaciales}

Nous supposons dans cette première approche que les surfaces de contact sont constantes aux deux interfaces électrode/tôle et tôle/tôle et égales à la surface de la face active de l'électrode, simplifiant de fait l'aspect macroconstriction.

En revanche, concernant les effets de microconstriction, nous tentons de rendre compte au mieux, des résistances de contact électrique et thermique et de leurs évolutions avec l'élévation de température durant le soudage en intégrant les valeurs de $R C E$ et $R C T$ obtenues expérimentalement. D'autres paramètres importants doivent être précisés. Il s'agit de la température d'interface, qui peut dépendre en particulier des températures des surfaces en contact, et d'un coefficient de partage de la puissance dissipée par effet Joule dans la résistance électrique interfaciale. Les études concernant ces deux paramètres sont actuellement peu nombreuses et pas encore abouties. Sur la base de travaux récents $[7,10]$ on retient ici la valeur de 0,8 pour le coefficient de partage et on identifie la température de l'interface à celle côté tôle. 


\subsubsection{Propriétés thermo-électriques et thermo-physiques}

Les propriétés électriques des matériaux (conductivité) et les propriétés thermophysiques (conductivité thermique, masse volumique, chaleur spécifique et enthalpie) constituent les différentes données d'entrée du modèle électrothermique. La résistivité électrique de l'acier soudé est une des principales données d'entrée et certainement la plus influente. PSA à l'issue d'une collaboration avec le Laboratoire de Génie Électrique de Paris (LGEP), s'est doté d'un moyen de mesure des propriétés électriques des matériaux en fonction de la température. Ce dispositif a permis la détermination de la résistivité électrique d'éprouvettes en acier en fonction de la température [7]. Les propriétés thermo-physiques sont toutes extraites de la thèse de Thiéblemont [8].

\subsubsection{Transformations métallurgiques}

Dans notre cas, les transformations métallurgiques à décrire sont principalement la transformation austénitique au chauffage, les transformations ferritique, bainitique et martensitique au refroidissement. Différentes approches peuvent être utilisées pour décrire les transformations de phase des aciers [24]. Les modèles peuvent être classés en deux groupes selon qu'ils s'appuient, pour leur identification, sur les cinétiques de transformation dans des conditions isothermes (diagrammes TTT : Temps, Température, Transformation) ou anisothermes (diagrammes TRC : Transformation en Refroidissement Continu) pour reproduire les transformations au refroidissement.

Les approches reposant sur des cinétiques isothermes ont surtout été développées à l'origine pour des applications de traitement thermique. Pour les applications de soudage, les conditions opératoires sont telles que l'on est dans une situation de chauffage rapide. Dès lors, des modèles s'appuyant sur les diagrammes TRC ont été proposés. Ces modèles reposent généralement sur une équation différentielle du type :

$$
\dot{p}=f(p, T, \ldots)
$$

où $p$ représente la proportion de phase formée.

Leblond [25] propose d'utiliser une simple équation différentielle du premier ordre :

$$
\dot{p}=\frac{\bar{p}(T)-p}{\tau(T)}
$$

où $\bar{p}(T)$ et $\tau(T)$ représentent deux paramètres, fonctions de la température, à ajuster pour chaque transformation pour retrouver le diagramme TRC. Il a été étendu au cas de plusieurs transformations entre plusieurs phases. Concernant la transformation martensitique, elle est indépendante du temps. La proportion de martensite formée $p_{M}$ ne dépend que de la température et est bien décrite par la loi de Koistinen-Marbürger [26] :

$$
p_{M}=p_{A} \cdot(1-\exp (-k(M s-T))) \text { pour } T \leq M s
$$

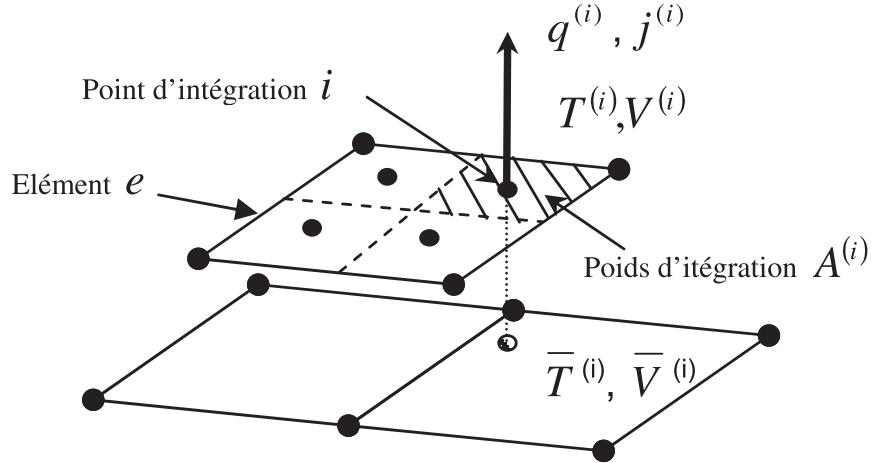

Fig. 8. Principe du contact électrothermique.

où $p_{A}$ représente la proportion d'austénite restant à transformer, $M s$, la température de début de transformation et $k$, un paramètre.

Les paramètres métallurgiques sont issus d'une étude PSA-SOLLAC-ESI Group [27]. Pour l'acier considéré (purement ferritique), 4 phases sont envisagées : Austénite (A), Ferrite (F), Martensite (M) et Bainite (B). Les phases $\mathrm{M}$ et $\mathrm{B}$ considérées simulent, dans ce cas, les phases plus dures rencontrées sur cet acier à très bas taux de carbone. Les effets de taille de grains sur les paramètres des cinétiques métallurgiques ne sont pas pris en compte.

\subsection{Modèle électro-thermo-métallurgique et mécanique}

L'approche précédente ne prend pas en compte l'influence des déformations mécaniques sur la géométrie du procédé et, en particulier, sur l'évolution des surfaces de contact. La prise en compte de tels phénomènes implique un couplage avec la mécanique [27].

La méthode des éléments finis repose sur une discrétisation des électrodes et des tôles. Par conséquent, chaque surface de contact électro-thermique est constituée d'éléments finis pouvant être de forme et de taille différente. Afin de modéliser le contact électro-thermique entre 2 maillages incompatibles, une formulation élément fini a été développée [28]. Les conditions de contact aux interfaces électrode-tôle et tôle-tôle sont traitées en chaque point de Gauss de chaque élément fini.

Cette méthode est présentée sur la figure 8 pour un élément d'une surface. Dans un premier temps, des densités de flux thermique et de courant électrique sont estimées à tous les points de Gauss de chacune des surfaces de contact et, dans un second temps, une correction est apportée de façon à assurer la conservation du flux thermique échangé ainsi que du courant. La description des conditions de contact porte sur deux surfaces de contact $S^{1}$ et $S^{2}$ en vis-à-vis appartenant à une électrode ou à une tôle.

Soient $A^{(i)}, T^{(i)}$ et $V^{(i)}$ la surface, la température et le potentiel électrique d'un point d'intégration $i(i=1, \ldots, n i)$ appartenant à un élément quelconque. 
Soient $\bar{T}^{(i)}$ et $\bar{V}^{(i)}$ la température moyenne et le potentiel moyen de l'élément de la surface opposée sur lequel se projette le point d'intégration $i$.

Soient $q^{(i)}$ et $j^{(i)}$ la densité de flux de chaleur et la densité de courant électrique associée au point d'intégration $i$.

$q^{(i)}$ peut être décomposé en deux parties :

$$
q^{(i)}=\varphi^{(i)}+p^{(i)}
$$

où $\varphi^{(i)}$ représente la densité de flux de chaleur échangée entre les surfaces $S^{1}$ et $S^{2}$, et $p^{(i)}$, une fraction de la densité de puissance dissipée à l'interface par effet Joule.

Nous supposons que l'interface ne présente aucune inertie thermique, la densité de flux de chaleur échangée $\varphi^{(i)}$ peut alors s'écrire de la façon suivante :

$$
\varphi^{(i)}=\frac{1}{R C T} \cdot\left(T^{(i)}-\bar{T}^{(i)}\right)
$$

où $R C T$ représente la résistance de contact thermique.

De la même façon, la densité de courant $j^{(i)}$ peut s'exprimer à partir de la résistance de contact électrique $R C E$ sous la forme :

$$
j^{(i)}=\frac{1}{R C E} \cdot\left(V^{(i)}-\bar{V}^{(i)}\right)
$$

La totalité de la densité de puissance dissipée par effet Joule à l'interface peut être exprimée à partir de (16) :

$$
\frac{1}{R C E} \cdot\left(V^{(i)}-\bar{V}^{(i)}\right)^{2}
$$

On suppose que la surface $S^{1}$ reçoit une fraction $\alpha^{1}$ de cette puissance dissipée à l'interface, alors que la surface $S^{2}$ reçoit une fraction $\alpha^{2}=1-\alpha^{1}$. Par conséquent, seule une partie $p^{(i)}$ doit être considérée au point d'intégration $i$ :

$$
p^{(i)}=\frac{\alpha^{(i)}}{R C E} \cdot\left(V^{(i)}-\bar{V}^{(i)}\right)^{2}
$$

Finalement, les équations (14), (15) et (18) donnent :

$$
q^{(i)}=\frac{1}{R C T} \cdot\left(T^{(i)}-\bar{T}^{(i)}\right)+\frac{\alpha^{(i)}}{R C E} \cdot\left(V^{(i)}-\bar{V}^{(i)}\right)^{2}
$$

Compte tenu de la forme des expressions locales de la densité de courant et de la densité de flux de chaleur adoptée, une correction doit être apportée. En effet, cette modélisation locale au point d'intégration n'assure pas nécessairement la conservation globale du flux thermique et du courant :

$$
\begin{aligned}
\int_{S_{1}} j \cdot \mathrm{d} s+\int_{S 2} j \cdot \mathrm{d} s & =\sum_{S_{1}} j^{(i)} \cdot A^{(i)}+\sum_{S_{2}} j^{(i)} \cdot A^{(i)} \\
& =I^{S 1}+I^{S 2} \neq 0 \\
\int_{S_{1}} \varphi \cdot \mathrm{d} s+\int_{S_{2}} \varphi \cdot \mathrm{d} s & =\sum_{S_{1}} \varphi^{(i)} \cdot A^{(i)}+\sum_{S_{2}} \varphi^{(i)} \cdot A^{(i)} \\
& =\phi^{S 1}+\phi^{S 2} \neq 0
\end{aligned}
$$

Deux coefficients de correction $C t$ et $C e$ respectivement associés aux problèmes thermique et électrique sont introduits de la façon suivante :

$$
\begin{aligned}
& C e=\sqrt{-\frac{I^{S 2}}{I^{S 1}}} \\
& C t=\sqrt{-\frac{\phi^{S 2}}{\phi^{S 1}}}
\end{aligned}
$$

À partir des équations (20) et (21), Ct et $C e$ permettent d'assurer les équilibres thermique et électrique :

Pour la surface de contact $S^{1}$ :

$$
j^{(i)^{*}}=C e \cdot \frac{1}{R C E} \cdot\left(V^{(i)}-\bar{V}^{(i)}\right)
$$

$$
\begin{aligned}
q^{(i)^{*}}=C t \cdot \frac{1}{R C T} \cdot & \left(T^{(i)}-\bar{T}^{(i)}\right) \\
& +C e^{2} \cdot \frac{f^{(i)}}{R C E} \cdot\left(V^{(i)}-\bar{V}^{(i)}\right)^{2}
\end{aligned}
$$

Pour la surface de contact $S^{2}$ :

$$
j^{(i)^{*}}=\frac{1}{C e} \cdot \frac{1}{R C E} \cdot\left(V^{(i)}-\bar{V}^{(i)}\right)
$$

$$
\begin{aligned}
q^{(i)^{*}}=\frac{1}{C t} \cdot \frac{1}{R C T} & \left(T^{(i)}-\bar{T}^{(i)}\right) \\
& +\frac{1}{C e^{2}} \cdot \frac{f^{(i)}}{R C E} \cdot\left(V^{(i)}-\bar{V}^{(i)}\right)^{2}
\end{aligned}
$$

\section{Validation expérimentale des modèles numériques}

Le développement d'un modèle doit s'accompagner d'une validation expérimentale pertinente des résultats numériques. Dans le cadre du soudage par point, la brièveté et le confinement de la formation de la soudure rendent délicate l'obtention de telles données. Dans la littérature, la majorité des études se cantonne à des mesures post-soudage, plus simples à obtenir, mais qui ne renseignent que sur l'état final de la soudure. D'autre part, nombre de ces travaux exposent des cas de soudage parfois trop complexes pour qu'il soit possible de procéder à une analyse critique des résultats.

Nous proposons de valider nos modèles par confrontation des cycles thermiques simulés à des cinétiques thermiques enregistrées dans l'assemblage durant l'exécution de points soudés (mesures dans les tôles et dans les électrodes). Les configurations de soudage étudiées se limitent à des assemblages symétriques (deux tôles identiques de même nature et de même épaisseur) et au cas académique de la tôle équivalente d'épaisseur double dont la comparaison au cas «classique » de soudage est riche en enseignements. Les tôles considérées dans l'étude sont en acier extra-doux et non revêtues. 


\subsection{Techniques d'instrumentation d'assemblage de tôles et d'électrodes}

Dans l'objectif double de valider nos modèles et d'identifier par méthodes inverses, à terme, les propriétés interfaciales, nous avons choisi d'instrumenter en capteurs de température l'électrode et les tôles [29]. Les techniques d'instrumentation ont été développées et réalisées au LET2E de l'Université Bretagne Sud de Lorient [7]. Par le recours à des microcapteurs et en optimisant les différents usinages et perçages des pièces, les méthodes d'instrumentation sont développées de manière à être les moins intrusives possibles. L'électrode est ainsi percée par des micro-forets à différentes distances de son axe et de sa face active. Dans les tôles, des usinages réalisés au moyen de fraises miniatures permettent d'aménager des petits puits pour la soudure chaude du thermocouple, soudée par décharge capacitive à l'aide d'une micro-électrode de cuivre. Les fils sont alors dégagés de la zone de soudage dans des rainures usinées à la surface des tôles.

En particulier, des assemblages de tôles nues, en acier extra-doux de 1,5 mm d'épaisseur, ont été instrumentés : dans l'électrode (diamètre face active $8 \mathrm{~mm}$ ), sept thermocouples de diamètre $240 \mu \mathrm{m}$ (type K, Thermocoax ${ }^{\circledR}$ ), gainés en acier inoxydable, sont introduits dans des puits de $0,25 \mathrm{~mm}$ de diamètre situés dans trois plans transverses à différentes distances de l'interface électrode/tôle $(0,5,1,0$ et $6,5 \mathrm{~mm})$. Les soudures des thermocouples, réparties sur différents diamètres $(1,0,3,0,5,0$ 6,0 et $7,0 \mathrm{~mm})$ sont plaquées mécaniquement au fond de leur trou. Les deux tôles sont instrumentées en thermocouples de type $\mathrm{K}$ au niveau des interfaces électrode/tôle et tôle/tôle. Quinze thermocouples de $50 \mu \mathrm{m}$ sont ainsi soudés par décharge capacitive à $0,5 \mathrm{~mm}$ de profondeur et répartis sur différents diamètres (de 1,0 à 10,0 mm). Les boules des thermocouples sont préalablement aplaties et logées dans les trous fraisés, tandis que les fils sont dégagés dans des rainures de 0,3 $\mathrm{mm}$ de profondeur.

L'ensemble des essais est réalisé sur des éprouvettes normalisées au moyen d'une presse de soudage implantée au LGM (Polytech'Nantes). L'acquisition des températures est réalisée à la fréquence de $1 \mathrm{kHz}$ par une centrale IOTECH ${ }^{\circledR}$, équipée d'une carte thermocouple DBK19.

Les matériaux utilisés ici sont des aciers extra-doux non revêtus (XES). Les paramètres de soudage sont définis sur la base de la norme E34.03.180.G [16].

\subsection{Confrontation des résultats numériques aux données expérimentales}

6.2.1 Application du modèle simplifié au soudage de deux tôles XES de $1,0 \mathrm{~mm}$ et d'une tôle équivalente d'épaisseur double

Le modèle simplifié est appliqué aux cas de soudage d'un assemblage de deux tôles XES de 1,0 mm d'épaisseur et d'une tôle équivalente de 2,0 $\mathrm{mm}$. Le recours au cas académique de la tôle d'épaisseur double nous permet
Tableau 1. Comparaison des résultats expérimentaux et simulés - assemblage de deux tôles XES de 1,0 mm d'épaisseur ou de la tôle équivalente d'épaisseur double.

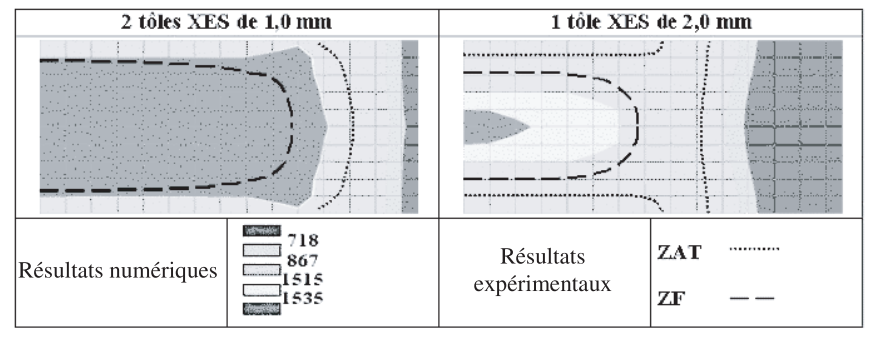

de découpler les effets des interfaces électrode/tôle et tôle/tôle.

Le tableau 1 compare les points soudés simulés et expérimentaux obtenus sur un assemblage de deux tôles XES de 1,0 mm d'épaisseur et sur une tôle équivalente de $2,0 \mathrm{~mm}$.

Les paramètres de soudage sont : $E=270$ daN, $I=$ $8,8 \mathrm{kA}$ et $t=10+10 \sim$. Les essais de soudage ont été réalisés avec une électrode neuve.

\subsubsection{Cas de la tôle équivalente d'épaisseur double}

À l'image des calculs réalisés avec le jeu de données de Thiéblemont [8], le modèle ne permet pas de développer une zone fondue de taille satisfaisante sur une tôle nue d'épaisseur double. Ce comportement résulte soit d'une mauvaise prise en compte des phénomènes de microconstriction à l'interface électrode/tôle (couple $R C E / R C T$ ), soit d'une sous-estimation des effets de macroconstriction à cette même interface. Or, les valeurs de $R C E$ (électrode/tôle) et $R C T$ (électrode/tôle) sont en assez bonne adéquation avec la littérature. En revanche, les résultats de la simulation de la phase d'accostage ont mis en évidence, qu'au début de la phase de soudage, le rayon de contact à l'interface électrode/tôle n'a pas atteint la valeur de celui de la face active de l'électrode $(1,8 \mathrm{~mm}$ contre $3 \mathrm{~mm}$ avec des électrodes neuves). Aussi, la macroconstriction du courant électrique résultant de ce fait n'est pas pris en compte et pourrait expliquer les mauvais résultats.

\subsubsection{Cas de l'assemblage de deux tôles simples}

Dans ce cas, comme ce que nous avions déjà obtenu avec les valeurs de Thiéblemont [8], le noyau est fortement surestimé. Toutefois, cette zone fondue excessive résulte vraisemblablement des valeurs de résistance de contact électrique tôle/tôle trop élevées, qui compensent les effets de macrosconstriction du courant à cette interface. Le calcul mécanique simulant la phase d'accostage montre d'ailleurs que le rayon de contact à l'interface (tôle/tôle) vaut seulement $1,8 \mathrm{~mm}$ à l'issue de la mise en pression, avec des électrodes neuves. 


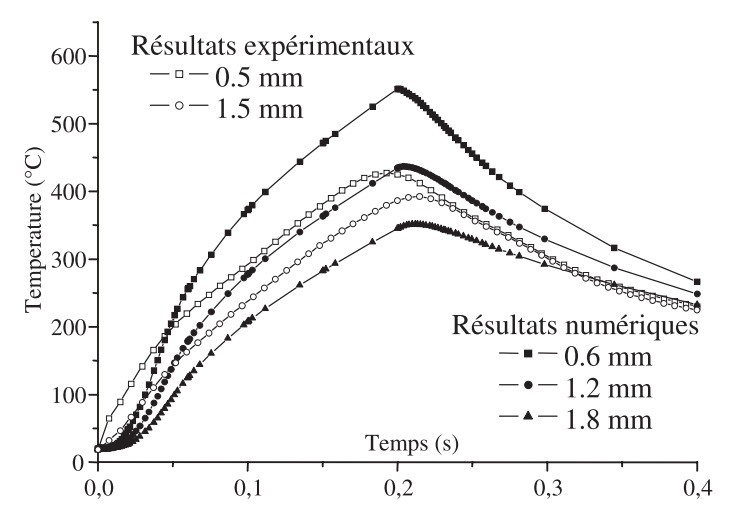

Fig. 9. Comparaison des cinétiques thermiques mesurées et calculées dans l'électrode sur l'axe d'axisymétrie de l'assemblage, pour le soudage de deux tôles XES d'épaisseur $1 \mathrm{~mm}$ (les distances correspondent à l'éloignement de l'interface électrode/tôle).

Cela conduit dans les premiers instants à des niveaux thermiques prédits dans la tôle irréalistes. S'agissant des cinétiques thermiques calculées dans l'électrode (Fig. 9), leur allure au chauffage est assez conforme à celle convexe expérimentale, en accord avec la très forte énergie dégagée initialement à l'interface tôle/tôle. Par contre, il apparaît un gradient axial de températures maximales trop important.

Cette confrontation montre toute la pertinence de disposer de cinétiques thermiques pour valider correctement le modèle numérique. Elle met également en exergue la difficulté d'une modélisation fidèle des conditions interfaciales. Pour la simulation du soudage d'un assemblage de tôles nues avec des électrodes neuves, nous confirmons que la prise en compte de l'évolution des rayons de contact aux interfaces est a priori nécessaire, l'application du modèle simplifié à ce cas ayant montré ses limites.

\subsubsection{Application du couplage mécanique au soudage de deux tôles XES de $1,5 \mathrm{~mm}$}

Nous avons choisi un cas de soudage sur tôles épaisses, configuration qui nécessite le recours à des électrodes dont le diamètre de la face active est de $\Phi 8 \mathrm{~mm}$. Nous supposons que le plus grand rayonnement de la face active ( $R=50 \mathrm{~mm}$, contre $R=40 \mathrm{~mm}$ pour une électrode $\Phi 6 \mathrm{~mm})$ augmente l'effet de macroconstriction du courant de soudage aux interfaces, rendant de fait plus pertinent encore un traitement mécanique du problème pour la prise en compte de la variation du rayon aux interfaces.

Les évolutions des résistances de contact électrique et thermique intégrées dans ce modèle sont celles proposées par Thiéblemont. Les valeurs des paramètres du macro-élement de contact électro-thermique au contact électrode/tôle sont, 0,8 pour le coefficient de partage et la température côté tôle pour la température de l'interface.

Le tableau 2 permet la comparaison des points soudés simulés et expérimentaux obtenus sur un assemblage de deux tôles XES de 1,5 mm d'épaisseur.
Tableau 2. Comparaison des résultats expérimentaux et simulés - assemblage de deux tôles XES de 1,5 mm d'épaisseur.
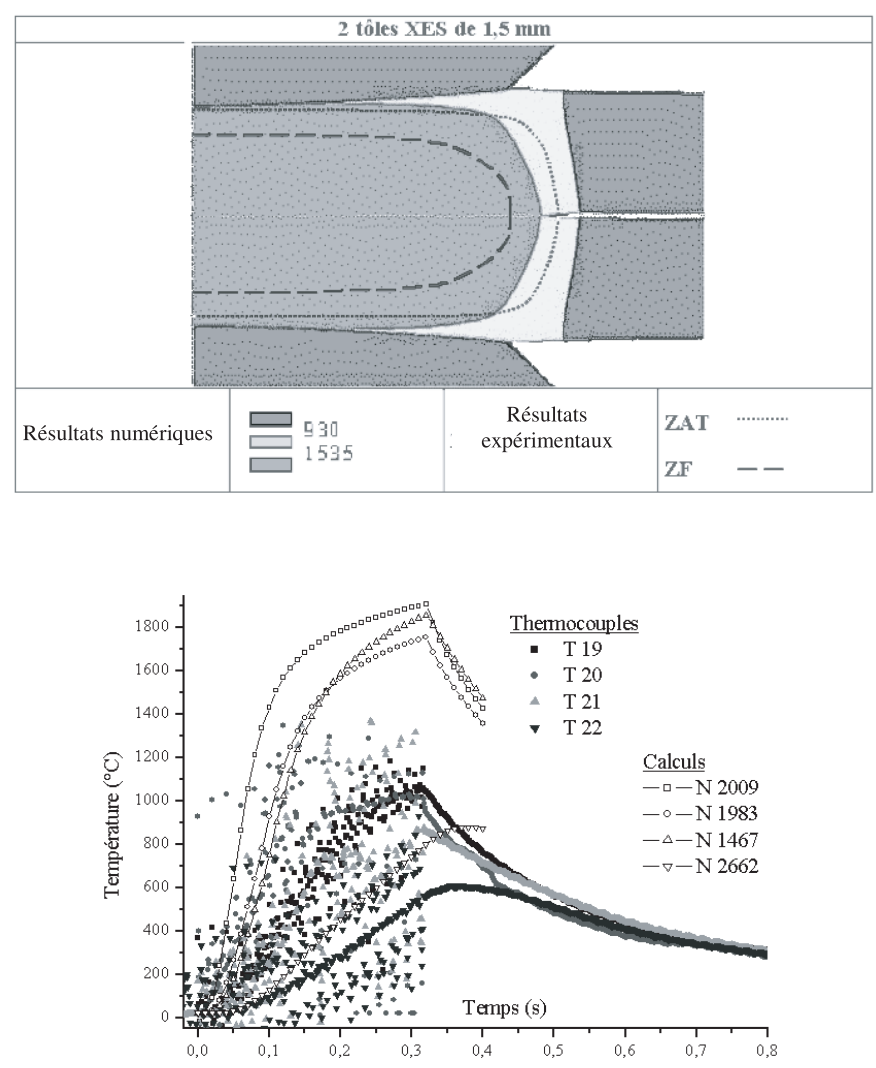

Fig. 10. Comparaison des cycles thermiques prédits et expérimentaux dans les tôles (assemblage de tôles XES de $1,5 \mathrm{~mm})$.

Les paramètres de soudage sont : $E=400$ daN, $I=$ $10,6 \mathrm{kA}$ et $t=16+14 \sim$. Les électrodes sont neuves lors des essais de soudage.

La comparaison calculs/expériences fait apparaître une surestimation de la taille du noyau simulé. En fait, la prise en compte de la macroconstriction associée à une microconstriction mal définie peut expliquer cette surchauffe des tôles. Dans la simulation, le noyau apparaît d'ailleurs beaucoup trop tôt par rapport à l'expérience (nous avons ainsi : $t_{\text {fus_calcul }}<0,01 \mathrm{~s}$ contre $\left.t_{\text {fus_exp }}=0,08 \mathrm{~s}\right)$. La fusion est générée dans les premiers instants par la source surfacique à l'interface tôle/tôle, qui résulte de l'action combinée des valeurs de la résistance de contact électrique tôle/tôle et des fortes densités de courant électrique liées à l'effet de macroconstriction à cette interface.

L'incohérence des résultats numériques peut provenir, d'une part, d'une prise en compte insuffisante de tous les phénomènes à l'origine de la variation des rayons de contact et, d'autre part, des valeurs de résistances de contact électriques excessives à l'interface tôle/tôle.

Les figures 10 et 11 comparent respectivement des cycles thermiques expérimentaux dans la tôle et dans l'électrode à des cycles thermiques simulés en des nœuds proches de la localisation des thermocouples. Les 


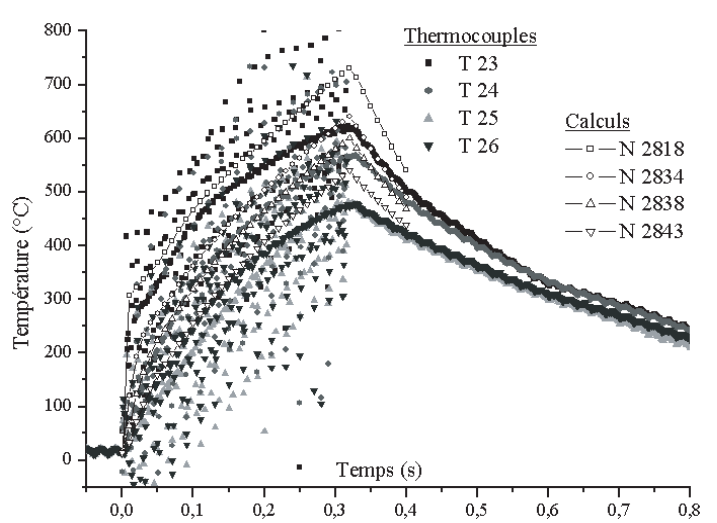

Fig. 11. Comparaison des cycles thermiques prédits et expérimentaux dans les électrodes (assemblage de tôles XES de $1,5 \mathrm{~mm})$.

Tableau 3. Cotes des points de mesure, par rapport au centre de l'assemblage, dans les tôles et des nœuds du maillage - tôles XES de $1,5 \mathrm{~mm}$.

\begin{tabular}{ccc}
\hline $\begin{array}{c}\text { Nouds/ } \\
\text { Thermocouples }\end{array}$ & $\begin{array}{c}\text { Cote radiale } \\
(\mathrm{mm})\end{array}$ & $\begin{array}{c}\text { Cote axiale } \\
(\mathrm{mm})\end{array}$ \\
\hline N 2009 & 3,0 & 1,0 \\
N 1983 & 3,5 & 1,0 \\
N 1467 & 4,0 & 0,5 \\
N 2662 & 5,0 & 0,5 \\
T 19 & $3,5 \pm 0,1$ & $1,0 \pm 0,1$ \\
T 20 & $3,0 \pm 0,1$ & $1,0 \pm 0,1$ \\
T 21 & $4,0 \pm 0,1$ & $0,5 \pm 0,1$ \\
T 22 & $5,0 \pm 0,1$ & $0,5 \pm 0,1$ \\
\hline
\end{tabular}

Tableau 4. Cotes des points de mesure, par rapport au centre de l'assemblage, dans les électrodes et des nœuds du maillage - tôles XES de 1,5 mm.

\begin{tabular}{ccc}
\hline $\begin{array}{c}\text { Nouds/ } \\
\text { Thermocouples }\end{array}$ & $\begin{array}{c}\text { Cote radiale } \\
(\mathrm{mm})\end{array}$ & $\begin{array}{c}\text { Cote axiale } \\
(\mathrm{mm})\end{array}$ \\
\hline N 2818 & 0,5 & 2,0 \\
N 2834 & 2,5 & 2,0 \\
N 2838 & 3,0 & 2,0 \\
N 2843 & 3,5 & 2,0 \\
T 23 & $0,5 \pm 0,05$ & $2,0 \pm 0,05$ \\
T 24 & $2,5 \pm 0,05$ & $2,0 \pm 0,05$ \\
T 25 & $3,0 \pm 0,05$ & $2,0 \pm 0,05$ \\
T 26 & $3,5 \pm 0,05$ & $2,0 \pm 0,05$ \\
\hline
\end{tabular}

tableaux 3 et 4 précisent les cotes des nœuds du maillage et des points d'implantation des thermocouples.

La comparaison des cycles thermiques dans les tôles confirme la taille excessive du noyau simulé. Les niveaux de température ainsi que les vitesses thermiques calculées sont largement supérieures aux résultats expérimentaux. Nous retrouvons d'ailleurs la surestimation de la taille du noyau pour les nœuds du maillage où la température de fusion est dépassée alors que la température des thermocouples correspondant indique qu'ils sont bien localisés en ZAT.

A contrario, dans l'électrode, les niveaux thermiques prédits par le calcul sont comparables à l'expérience.
L'utilisation des valeurs de Thiéblemont conduit au paradoxe suivant : malgré une forte surchauffe des tôles, les électrodes présentent des températures équivalentes aux mesures expérimentales. Cette incohérence provient certainement d'une valeur de résistance de contact thermique largement surestimée à l'interface électrode/tôle, qui entrave la diffusion du flux de chaleur vers l'embout de soudage.

La mise en œuvre d'un modèle plus complet traitant de la variation des rayons de contact ne permet pas non plus d'obtenir pour l'instant des résultats satisfaisants. La grande difficulté à modéliser correctement tous les phénomènes à l'origine de la variation des rayons de contact (définition et caractérisation des lois de comportement mécaniques à chaud, choix de la condition mécanique de contact...) peut expliquer en partie la discordance.

\section{Conclusion}

L'objet de cette étude était d'opérer une « remise à plat » du soudage par point afin d'expliquer les phénomènes physiques à l'origine de la soudure ainsi que leur rôle et leur importance. En raison de la complexité du procédé de soudage et du couplage des phénomènes impliqués, nous avons choisi de travailler sur des configurations simples en nous limitant aux assemblages symétriques d'aciers extra-doux et au cas académique de la tôle équivalente d'épaisseur double, qui présente l'avantage de supprimer l'interface tôle/tôle.

Pour répondre à cet objectif, 2 modèles numériques ont été proposés : un modèle simplifié dans lequel on a supposé les surfaces de contact aux interfaces électrodetôle et tôle-tôle constantes, et un modèle plus élaboré dans lequel les évolutions des surfaces de contact au cours des différentes phases du procédé de soudage (accostage, soudage et forgeage) sont prises en compte. Le premier modèle a permis de confirmer le rôle essentiel des conditions de contact aux interfaces électrode/tôle et tôle/tôle sur le procédé, au travers de leur influence sur la taille du noyau, sur sa cinétique de formation, ainsi que sur les cycles thermiques dans les tôles et dans les électrodes. La formation d'un point soudé est donc fortement dépendante des phénomènes aux interfaces, qui se manifestent à deux échelles : à l'échelle microscopique dans l'évolution des résistances électrique et thermique de contact, et à l'échelle macroscopique dans l'évolution de l'aire des surfaces de contact. Ces surfaces varient de façon complexe et sont affectées par la configuration de l'assemblage (tôles nues ou revêtues, épaisseur, paramètres de soudage... ), et par le profil de la face active des électrodes (plat ou rayonné, neuf ou dégradé...).

Avec la répétition des soudures, l'analyse expérimentale a montré que le profil des embouts de soudage, initialement rayonné, présentait rapidement un méplat dont la taille augmentait avec le nombre de soudures. La modélisation de la phase d'accostage pour des assemblages à embout $\Phi 6 \mathrm{~mm}$ a montré alors que les surfaces de contact aux interfaces électrode/tôle 
et tôle/tôle, en considérant dans le modèle un profil d'électrode usagée, approchaient $3,0 \mathrm{~mm}$, rayon de la face active de l'électrode.

Nous avons présenté un dispositif ex-situ de caractérisation des résistances de contact électrique et thermique, en fonction de la température (jusqu'à $450{ }^{\circ} \mathrm{C}$ ) et sous pression (jusqu'à $40 \mathrm{MPa}$ ). Une méthodologie de mesure originale, faisant appel à une technique d'empilement d'échantillons, ainsi qu'un protocole spécifique de préparation des éprouvettes, ont accompagné la conception du dispositif de mesure. On a ainsi déterminé un jeu de données complet des résistances de contact électrique et thermique en fonction de la température notamment pour des tôles nues.

Dans l'objectif double de valider nos modèles et d'identifier par méthodes inverses, à terme, les propriétés interfaciales, nous avons choisi d'instrumenter en capteurs de température l'électrode et les tôles. Par le recours à des micro-capteurs et en optimisant les différents usinages et perçages des pièces, les méthodes d'instrumentation ont été développées de manière à être les moins intrusives possibles. La réussite des campagnes d'essais de soudage successives, réalisées sur assemblages instrumentés en capteurs de température, atteste de la robustesse des techniques spécifiquement développées. Les instrumentations se sont révélées bien adaptées à l'environnement pourtant sévère et aux conditions délicates du procédé de soudage par point (fortes sollicitations mécaniques ainsi que rapidité du temps de soudage et confinement de la soudure). Les cinétiques thermiques obtenues dans les électrodes et surtout dans les tôles constituent des informations originales et très intéressantes pour la connaissance du procédé de soudage.

Au plan numérique, sur des tôles nues soudées avec des électrodes neuves, le modèle numérique simplifié est en défaut, l'hypothèse des surfaces de contact constants étant ici trop réductrice : ceci a pu être clairement mis en évidence en ayant recours au cas académique de la tôle équivalente d'épaisseur double qui élimine l'effet de l'interface tôle/tôle. Dans l'objectif de prendre en compte la variation des surfaces de contact, le recours à un modèle couplant les phénomènes électro-thermométallurgique et mécanique, n'a pas pour le moment conduit à des résultats satisfaisants. Ce calcul montre la difficulté à rendre compte fidèlement des phénomènes complexes qui sont à l'origine de l'effet de la macroconstriction du courant aux interfaces électrode/tôle et tôle/tôle. Dans les deux cas, l'analyse des résultats fait ressortir l'importance de l'évolution des résistances de contact aux interfaces. Dans le cadre du modèle simplifié et pour notre jeu de données de résistance de contact, la valeur de la résistance de contact électrique à l'interface tôle/tôle s'est révélé trop forte. Dans le cas du modèle thermomécanique, avec le jeu de données issu des travaux de Thiéblemont, on montre non seulement que la valeur de la résistance de contact électrique à l'interface tôle/tôle est excessive, mais aussi la résistance de contact thermique à l'interface électrode/tôle.
Remerciements. Nous souhaitons remercier Mrs Costa et Dumons du LET2E pour leur aide précieuse concernant l'instrumentation des assemblages et la mesure des résistances de contact.

\section{Références}

[1] H. Murakawa, J. Zhang, FEM simulation of spot welding process (report 1) - Effect of an initial gap on nugget formation, Trans JWRI 27 (1998) 75-82

[2] B.H. Chang, M.V. LI, Y. Zhou, Comparative study of small scale and "large scale" resistance spot welding, Mathematical modelling of weld phenomena VI, Graz, $1^{\mathrm{er}}-3$ octobre 2001, H. Cerjak (éd.), 923-937

[3] V. LI, M. Kimchi, Modeling of resistance spot welding high-strength steel, International Body Engineering Conference'00, Body Assembly \& Manufacturing, Vol. 34, Detroit, 3-5 October 2000

[4] P.S. James, H.W. Chandler, J.T. Evans, J. Wen, D.J. Browne, C.J. Newton, The effect of mechanical loading on the contact resistance of coated aluminium, Materials Science and Engineering A230 (1997) 194-201

[5] S.S. Babu, M.L. Santella, Z. Feng, B.W. Riemer, J.W. Cohron, Empirical model of effects of pressure and temperature on Electrical Contact Resistance of metals, Science and Technology of Welding and Joining 6 (2001) 126-132

[6] M. Vogler, S. Sheppard, Electrical contact resistance under high loads and elevated temperatures, Welding J. 72 (1993) 231-298

[7] G. Sibilia, Modélisation du soudage par point, Influence des conditions interfacia. (a) Principe du soudage par résistance. (b) Exemple de Zone Fondue sur le procédé, Thèse, Polytechnique Nantes, 2003

[8] E. Thieblemont, Modélisation du soudage par résistance par points, Thèse, INPL, 1992

[9] J-J. Salgon, O. Quemener, M. Belghali, J. Bransier, Résistance thermique de contact statique. Évaluation expérimentale d'un modèle à deux résistances issu d'une description probabiliste des déformations de l'interface, Revue générale de Thermique 37 (1998) 284-294

[10] G. Le Meur, Étude de la condition de liaison thermique à une interface de contact solide-solide siège d'une dissipation par effet Joule : application au soudage par point, Thèse, Université de Nantes, 2002

[11] Rapport DETB 7101, Rapport des principales recherches sur les résistances thermiques de contact, Université de Nantes, Département d'échanges thermiques, Laboratoire de Thermocinétique, 1971

[12] Z. Feng, S.S. Babu, M.L. Santella, B.W. Riemer, J.E. Gould, An incrementally coupled Electrical-thermalmechanical model for Resistance Spot Welding, 5th International Conference on Trends in Welding Research, Pine Mountain, 1-5 juin 1998

[13] T. Dupuy, La dégradation des électrodes lors du soudage par points de tôles zinguées, Thèse, ENSMP, 1998

[14] P. Dong, M.V. Li, M.Kimchi, Finite Element analysis of electrode wear mechanisms : face extrusion and pitting effects, Science and Technology of Welding and Joining 3 (1998) 59-64

[15] P. Dong, F. Lu, Model for estimating electrode face diameter during resistance spot welding, Science and Technology of Welding and Joining 4 (1999) 285-289 
[16] E34.03.180.G, Norme PSA Peugeot Citroën, Soudage électrique par résistance : Techniques de soudage, Spécifications générales, mars 2001

[17] O.P. Gupta, A. De, An improved numerical Modeling for resistance spot Welding process and its experimental verification, J. Manufacturing Science and Technology 120 (1998) 246-251

[18] D.J. Browne, H.W. Chandler, J.T. Evans, J. Wen, Computer simulation of resistance spot welding in aluminium : Part I, Welding J. 74 (1995) 339-344

[19] J.A. Khan, L. Xu, Y.-J. Chao, K. Broach, Numerical simulation of resistance spot welding process, Numerical Heat Transfer Part A 37 (2000) 425-446

[20] M.V. Li, P. Dong, M. Kimchi, Modeling of contact resistance during resistance spot welding, NIST Special publication 923 (1997) 423-434

[21] E. Thieblemont, J.V.C. Chevrier, P. Conraux, J.-M. Bergheau, P. Gobez, Couplage électro-thermique. Application au soudage par résistance par point, Actes STRUCOME, Paris, 1991, pp. 143-156

[22] SYSWELD ${ }^{\circledR}$, Manuel utilisateur, ESI Group 2003

[23] J. Negre, Le soudage électrique par résistance, Publications de la Soudure Autogène, $4^{\mathrm{e}}$ édition, 1972

[24] S. Denis, Prévision des contraintes résiduelles induites par traitement thermique et thermochimique, Revue de métallurgie - CIT/Science et Génie des matériaux, pp. 157-176 (1997)
[25] J.-B. Leblond, J.-C. Devaux, A new kinetic model for anisothermal transformation metallurgical transformations in steel including effect of austenite grain size, Acta Metallurgica, 32 (1984) 137-146

[26] D.P. Koistinen, R.E. Marburger, A general equation prescribing the extent of austenite-martensite transformation in pure iron carbon alloys and plain carbon steels, Acta Metallurgical 7 (1959) 417-426

[27] V. Robin, A. Sanchez, T. Dupuy, J. Soigneux, J.M. Bergheau, Numerical simulation of spot welding with special attention to contact conditions, Graz-Seggau, Austria, October 2001, H. Cerjak (eds.), Mathematical Modelling of Weld Phenomena 6, The Institute of Materials, London, 2002

[28] E. Feulvarch, V. Robin, J.M. Bergheau, Resistance Spot Welding Simulation : A General Finite Element Formulation of Electrothermal Contact Conditions, Proceedings of AMPT 2003, The International Conference on Advances in Materials and Processing Technologies, Dublin City University (Ireland), July 8-11, D.G. Olabi, S.J. Hashmi, 2003, pp. 558-561

[29] G. Sibilia, P. Rogeon, G. Saindrenan, Experimental validation of an electrical-thermal-metallurgic predictive model in resistance spot welding, 2nd International Conference on Thermal Process Modelling and Computer Simulation, Nancy, 31 mars-2 avril 2003

Retrouvez nos articles sur le site : www.edpsciences.org/meca 